\title{
Eine Neubewertung der „Gift Relationship“ in der britischen Geschichte zum Freiwilligensektor
}

George Campbell Gosling

Es gibt nicht die eine Geschichtsschreibung, die karitative Arbeit, Kampagnen, gemeinnützige Organisationen, Freiwilligenverbände, Zivilgesellschaft, den dritten Sektor und Nichtregierungsorganisationen behandelt. Stattdessen lassen sich vier zentrale Forschungszweige ausmachen, die diesen Bereich neuerer britischer Geschichte abdecken. Sie sind nicht klar voneinander zu trennen, und Historiker wechseln von einem Schwerpunkt zum anderen, aber sie gingen dabei unterschiedlichen Zielsetzungen und Fragestellungen nach. Dazu zählt erstens eine Sozialgeschichte, die sich in erster Linie mit wohltätiger Arbeit und Armutsbekämpfung befasst und diese in den größeren Kontext der Beziehungen zwischen Arm und Reich einordnet. Zweitens ist eine Geschichte der Freiwilligenarbeit zu nennen, die aus dem Wunsch der ehrenamtlich Engagierten heraus entstanden ist, ihre Arbeit historisch zu verorten und aus der eigenen kollektiven Geschichte zu lernen. Drittens gibt es eine politische Geschichte, die sich mit der Beziehung zwischen dem freiwilligen Sektor und dem Staat befasst. Dazu gehören auch Arbeiten, deren besonderes Interesse den Nichtregierungsorganisationen nach 1945 gilt, die sich also einer speziellen Kategorie freiwilliger Organisationen widmen, welche Lobbyarbeit und Interessensvertretung betreiben. Und schließlich haben wir eine Geschichte des Humanitarismus, welche die Begegnungspunkte zwischen Großbritannien und der weiteren Welt untersucht, die von imperialistischen und internationalistischen Impulsen ausgegangen sind, sei es durch Fundraising oder durch die Entsendung freiwilliger Helfer nach Übersee.

Ziel dieses Aufsatzes ist es nicht, einen Überblick über diese nebeneinanderherlaufenden Forschungszweige zu liefern. Vielmehr soll hier das Ungleichgewicht zwischen ihnen thematisiert werden. Dieses Ungleichgewicht ist eine Konsequenz der fruchtbaren Dynamik, die sich vor allem auf dem Feld der politischen Geschichte und der Geschichte des Humanitarismus entwickelt hat, die jedoch die Sozialgeschichte zu einem gewissen Grad in ihren Schatten stellt. Um das zu verstehen, werden wir zuerst die Anfänge dieses die Forschung zum freiwilligen Sektor prägenden Trends hin zur politischen Geschichte nachzeichnen, wobei dieser als Reaktion auf den Thatcherismus zu sehen ist. Anschließend wenden wir uns im Kontrast dazu der früheren Forschungsliteratur zur Sozialgeschichte und ihren theoretischen Schwerpunkten zu, um sodann zu analysieren, wie sich die politische Wende auf sie auswirkte. Insbesondere durch die Einbindung der Geschichte der Wohltätigkeit in die allgemeine Geschichte der Sozialfürsorge verschob sich der Schwerpunkt vom Konsum zum Angebot karitativer Wohlfahrt. Neuere kulturgeschichtliche Studien sprechen die älteren Fragen, die einst im 
Mittelpunkt der sozialgeschichtlichen Projektes lagen, dagegen meist nicht mehr an. Schließlich soll ein Ansatz vorgestellt werden, um dieses Projekt neu zu denken und zu beleben. Hierfür sollen Erkenntnisse aus der inzwischen gut etablierten Neuen Wirtschaftssoziologie hinzugezogen werden, die unserem Verständnis der Beziehung zwischen wirtschaftlichem und gesellschaftlichem Verhalten einen neuen Rahmen verliehen hat. Dieser Ansatz, wirtschaftliche Interaktion nicht nur im sozialen Kontext, sondern als eine Form sozialer Interaktion zu erforschen, eignet sich hervorragend für die Untersuchung von Wohltätigkeit - etwa indem das Verhältnis zwischen Spender und Empfänger in den Blick genommen wird.

Ob auf diesem oder anderem Wege: Es ist wichtig, einem neu konzipierten Programm für die Sozialgeschichte die Aufmerksamkeit zu schenken, die ihm gebührt. Dies nicht zu tun, hieße, einen fundamentalen Aspekt der Geschichte der Freiwilligenarbeit zu ignorieren: das Verhältnis zwischen Gebendem und Nehmenden, das im Englischen mit dem Begriff gift relationship bezeichnet wird. Und es hieße, ebenfalls eine Schlüsselfigur in dieser Geschichte auszublenden: den Empfänger. Einige herausragende Einzelstudien beleuchten inzwischen diesen Aspekt mithilfe neuer theoretischer Herangehensweisen, die, wie im Bereich der Sozialgeschichte allgemein, interdisziplinär ausgerichtet sind. Es fällt schwer, sich eine neue, dynamische Sozialgeschichte vorzustellen, die nicht passender als soziokulturelle Geschichte verstanden werden könnte. Diese innovativen Studien sind eher eine diffuse Sammlung als ein zusammenhängendes Gesamtprojekt. Dieser Aufsatz geht der Frage nach, wie man dies ändern kann und wie eine Neukonzeption des Wirtschaftsverhaltens als Sozialverhalten bei diesem Versuch nützlich sein könnte.

Es gibt kaum Bereiche der britischen Zeitgeschichte, auf die Margaret Thatcher keinen langen Schatten wirft. Ihre Amtszeit, die von 1979 bis 1990 dauerte, bezeichnet einen sichtbaren, wenn auch nicht immer sauberen Bruch mit der vorangegangenen Ära eines vermeintlichen Konsenses im Bereich der Wohlfahrtspolitik. Ihre Zeit als Premierministerin war für den freiwilligen Sektor in Großbritannien bedeutsam, wenn auch auf eine Weise, die nicht vollständig beabsichtigt war, und die wiederum tiefgreifende Auswirkungen darauf haben, wie wir die Geschichte der Wohltätigkeit verstehen und warum wir sie erforschen. ${ }^{1}$ Es darf behauptet werden, dass sie auf eine politische Wende in der Geschichte der Wohltätigkeit in Großbritannien hinausliefen.

Regierungen vor und nach Thatcher haben versucht, ehrenamtliche Tätigkeit zu fördern und ein engeres Arbeitsverhältnis zwischen Staat und ehrenamtlichem Sektor zu schmieden. Die Einrichtung 
der Voluntary Services Unit innerhalb des Innenministeriums (Home Office) unter der konservativen Regierung Edward Heaths, sowie der „Partnership“-Ansatz der Labour-Regierungen Tony Blairs sind Beispiele hierfür. ${ }^{2}$ Während der 1970er und 1980er setzten Thatcher und die Neue Rechte allgemein den Schwerpunkt auf nicht-staatliche Arten der Sozialfürsorge, und der Freiwillige Sektor ließ sich hier gut einspannen. ${ }^{3}$ Gegen Ende der 1980er wurden freiwillige Träger bei verschiedenen Regierungsprogrammen in den Bereichen des Wohnungs- und Arbeitsmarktes so behandelt, als seien sie mit kommerziellen Anbietern vollkommen austauschbar. Der Unterschied zwischen beiden war viel weniger wichtig als diejenige, die zwischen ihnen und dem Staat gezogen wurde. Es ließe sich annehmen, dass in Thatchers hoher Wertschätzung „viktorianischer Werte" der Glaube an die sozialen Vorzüge der Philanthropie auf einer Ebene mit ihrer antistaatlichen Einstellung zu sehen ist. Dies war jedoch nicht der Fall.

1977 sagte Thatcher in einer Rede, dass die „viktorianische Ära - die Glanzzeit der freien Marktwirtschaft in Großbritannien - ebenfalls die Epoche der Selbstlosigkeit und Wohltätigkeit darstellte“. ${ }^{4}$ Es war allerdings immer eindeutig, dass für sie das freie Unternehmertum an erster Stelle stand. Im Jahr 1988 sprach Thatcher vor der General Assembly der Church of Scotland, wo sie die theologischen Grundsätze ihrer Weltanschauung schilderte. Der Begriff "Wohltätigkeit“ fiel dabei nicht. Am nächsten kam sie noch mit folgender Wendung:

„Wie könnten wir auf die vielen Hilferufe reagieren, oder in die Zukunft investieren, oder die begabten Künstler und Handwerker unterstützen, deren Arbeit ebenso Gott preist, wenn wir nicht zuerst schwer gearbeitet und unsere Talente genutzt hätten, um den für diese Hilfe nötigen Wohlstand zu schaffen?"

Der inzwischen am häufigsten zitierte Satz aus dieser Rede lautet: „Im Christentum geht es um die spirituelle Erlösung, nicht um soziale Reformen." Thatchers Politik konzentrierte sich auf die Spannungen zwischen dem Staat und dem Bürger, aber ohne die sozialen Institutionen und Organisationen ernsthaft in Betracht zu ziehen, die zwischen beiden vermitteln. Wenn soziale Dienste für sie wichtig waren, dann als Bollwerk gegen einen übermächtigen Staat. Vor dem Women's Royal Volunteer Service sagte sie 1981, dass „die Bereitschaft von Männern und Frauen, ehrenamtliche Arbeit zu leisten, eine der größten Freiheitsgarantien ist. Sie stellt sicher, dass die Fürsorge von politischem Einfluss freibleibt. ${ }^{66}$ Freiwillige Organisationen waren nur insofern von Bedeutung, als sie für eine wirtschaftliche oder politische Zielsetzung eine Aufgabe erfüllten. Sie mussten nicht aktiv unterstützt werden, um die bürgerliche Gesellschaft als positiven Kontrast zu staatlicher Intervention und Sozialfürsorge zu entwerfen. Der zukünftige Premierminister Gordon Brown beobachtete 1988, noch als junger Oppositioneller, der im Schattenkabinett für das Finanzministerium vorgesehen war: 
„....wir haben gesehen, wie die Wohltätigkeit aus der Rumpelkammer der Sozialgeschichte herausgezogen und abgestaubt wurde und wie sie, zur Beschämung und Wut der Freiwilligenorganisationen Großbritanniens, einer skeptischen oder gleichgültigen Öffentlichkeit als unverzichtbarer Bestandteil eines durch und durch modernen Thatcherismus präsentiert wurde. ${ }^{17}$

Trotz Thatchers ambivalenter Haltung der Wohltätigkeit gegenüber vollzog sich während ihrer Amtszeit doch in dreierlei Hinsicht eine Veränderung in der Rolle, die dem freiwilligen Sektor zukam. Der Erste war die Zunahme der Freiwilligentätigkeit, und zwar insbesondere derjenigen, die mit dem Staat einherging und durch inn gefördert wurde. ${ }^{8}$ Der Wolfenden-Bericht von 1978 forderte noch vor Thatchers Amtsantritt und im Einklang mit Entwicklungen, die schon in den siebziger Jahren eingesetzt hatten, eine größere Bedeutung für private und ehrenamtliche Sozialfürsorge. Außerdem nahmen die finanziellen Mittel, welche die Regierung für den freiwilligen Sektor bereitstellte, zu. Solche staatlichen Fördergelder wuchsen von $£ 93$ Millionen in den Jahren 1979/80 auf $£ 293$ Millionen in 1987/8. ${ }^{9}$ In den 1980 Jahren änderte sich jedoch die Erwartungshaltung gegenüber karitativen Organisationen. Wie der Historiker Frank Prochaska feststellte, zentralisierten die Thatcher-Regierungen in ihrem Drang, den Staat einzudämmen, das, was von diesem übrigblieb, und vergrößerten so die Abhängigkeit des Freiwilligensektors von ihm. Bis heute wirft diese Entwicklung ernsthafte Fragen zur Unabhängigkeit des ehrenamtlichen Bereichs auf. Eliza Filby etwa hat über die Zusammenarbeit auf lokaler Ebene von Kirchen und Manpower Services Commission in den 1980er Jahren geschrieben, die den Arbeitslosen zwar einerseits praktische Hilfe leistete, aber andererseits die Kirchen in die Rolle paternalistischer Agenten des Staates drängte. Dies warf Probleme auf, als Änderungen der politischen Tagesordnung und die Streichung von Fördergeldern deutlich machten, dass freiwillige Organisationen für die Regierung nur eine niedrige Priorität hatten. Ihr Ruf litt dabei auch dadurch, dass sie mit einem unbeliebten politischen Plan assoziiert wurden. Vor allem anderen ist es daher als Thatchers nachhaltigstes Erbe in diesem Bereich anzusehen, dass sie eine zunehmende Politisierung wohltätiger Arbeit provozierte, die zweite der genannten Veränderungen.

Die dritte Veränderung vollzog sich innerhalb der ehrenamtlichen Organisationen selbst, indem sie sich professionalisierten. Wenngleich sich die Entwicklung professioneller Fähigkeiten und Standards in britischen Wohltätigkeitsorganisationen zwar bis ins frühe 20. Jahrhundert zurückverfolgen lassen, wird dieses Phänomen eher mit dem späten 20. Jahrhundert und sogar noch mehr mit der Wende zum 21. in Verbindung gebracht. ${ }^{10}$ "Allein die starke Zunahme in Größe und Ausmaß freiwilliger Einrichtungen bedeutete, dass viele, wenn nicht gar die meisten Organisationen, entschieden professioneller werden mussten. Dies führte sie anscheinend weit von ihren früheren aktiven Mitgliedern weg," stellten Matthew Hilton u.a. fest, und führten aus, wie mit der Aufstellung von 
„Geschäftsführern, Vertriebsdirektoren, Kampagnenleitern, Werbestrategien und wiedererkennbaren Markennamen“ im ehrenamtlichen Sektor die Möglichkeit einer "NGO-Karriere" geschaffen wurde. ${ }^{11}$ Wenn diese zunehmende Professionalisierung auch eher weitere Tendenzen wiedergab als die zielgerichtete Umsetzung eines politischen Programms, bereitete sie den Freiwilligensektor doch auf eine neue Ära vor, in welcher, wie der verstorbene Historiker Geoffrey Finlayson festhielt, der kommerzielle, der karitative und der informelle Sektor eher als „potenzielle Alternativen zum Wohlfahrtsstaat statt als Ergänzung oder Impuls dafür" betrachtet werden. ${ }^{12}$ Dadurch erhielt ehrenamtliche Tätigkeit eine neue politische Aktualität. Die Verschiebung der alten Wohlfahrtsbürokratien hin zu einer Vertragskultur, in der ehrenamtliche Agenturen im Wettbewerb mit öffentlichen und privaten Dienstleistern mitbieten konnten, begann zumindest in der Theorie schon vor 1979 und wurde bis 1990 weitergeführt sowie ausgeweitet. ${ }^{13}$ Während der Thatcher-Zeit war sie jedoch aktive Regierungspolitik. Nick Deakin hat darauf hingewiesen, dass die Ziele der Regierung in diesem Bereich in den 1980er Jahren darauf hinausliefen, die Philanthropie als Alternative zum Staat wiederzubeleben, und eine neue Motivation zur Übernahme von Verantwortung zu schaffen, die das Vertrauen auf den Staat ablösen sollte. Die Voluntary Services Unit sollte den Freiwilligensektor durch die Verbreitung der Konzepte des New Managerialism auf seine neuen Aufgaben vorbereiten. ${ }^{14}$

Unter den Praktikern und Forschern dieses erweiterten, professionalisierten und politisierten Freiwilligensektors erwuchs der Wunsch, sich mit dessen Geschichte zu befassen, was 1991 zur Gründung der Voluntary Action History Society (VAHS) führte. Gründungsmitglieder waren Justin Davis Smith, Rodney Hedley und Colin Rochester und auch Nick Deakin war früh involviert. Schon im Namen kam der bewusste Versuch zum Ausdruck, das Feld, wie es sich in der Gegenwart darbot, in der eigenen Geschichte zu verorten. Die Phrase „voluntary action“ war eine Referenz auf und an den wenig bekannten Bericht William Beveridges aus dem Jahr 1948. Beveridges viel prominenterer Bericht zur Sozialversicherung von 1942 lieferte den Entwurf für den britischen Wohlfahrtsstaat. Während der erste Bericht von der Kriegsregierung angefordert worden war, wurde der zweite Bericht vom National Council for Social Service in Auftrag gegeben, zu einem Zeitpunkt, als die LabourRegierung schon dabei war, die Empfehlungen Beveridges von 1942 weitgehend in die Tat umzusetzen. Während diese Empfehlungen zu einer umfassenden Erweiterung staatlicher Kompetenzen aufriefen, um die fünf größten Übel, die "five giants" (Not, Krankheit, Unwissen, Schmutz und Untätigkeit) in den Griff zu bekommen, erinnerte der zweite Bericht daran, dass in seiner Vision einer gerechteren Gesellschaft durchaus Raum für die Zivilgesellschaft blieb. Hierzu gehörte sowohl die Philanthropie, welche er als „den Impuls von oben“ bezeichnete, als auch die gegenseitige Hilfe, "den Impuls von unten“. ${ }^{15}$ Vier Jahrzehnte später und in einem stark veränderten Kontext wurde 
die VAHS ins Leben gerufen, da der Freiwilligensektor den Rückgriff auf die Geschichte nutzen wollte, um Halt zu finden und angesichts neuen Anforderungen entgegenzutreten, die in der Zeit nach Thatcher von den Regierungen an den Freiwilligensektor gestellt wurden.

Diese politisierte Geschichtsschreibung war die Priorität der Forscher zum und Praktiker im Freiwilligensektor, die ein Interesse an ihrer gemeinsamen Geschichte hatten. Sie scheint auch in den verschiedenen Aktivitäten desjenigen Historikers durch, der an den frühen Aktivitäten der VAHS am stärksten beteiligt war: Frank Prochaskas Arbeit besteht aus zwei Strängen: seiner eigenen empirischen Forschung zur Sozialgeschichte, die sich oftmals auf eine goldene Ära viktorianischer Philanthropie beruft, und der Arbeit für Think Tanks, welche die Unterschiede zwischen dieser Geschichte und der Gegenwart hervorhebt. ${ }^{16}$ So sehr Prochaskas Engagement in der zeitgenössischen Politik auch in seiner sozialgeschichtlichen Forschung wurzelte, die sich meist mit der Rolle der Frauen, der Religion und des Altruismus befasste, so wenig lässt sich diese Forschung als apolitisch bezeichnen. Tatsächlich bezeichnete Prochaska es in seinem Buch über Christentum und Sozialdienst im modernen Großbritannien als „ein Hauptanliegen“, den Einfluss des Wohlfahrtsstaates auf die Gesellschaft zu ermessen. ${ }^{17}$

In den letzten Jahren haben mit einem Forschungsverbund an der Universität Birmingham verbundene Historiker eine spürbare Dynamik in die Geschichte der Freiwilligenorganisationen gebracht, allen voran Matthew Hilton und Nick Crowson. Ihre eigene Forschung und die gemeinsame Arbeit für verschiedene Veranstaltungen und Publikationen stellt eine beträchtliche Herausforderung für Prochaskas Verfallsnarrativ dar. Hilton und Crowson betonen die Anpassungsfähigkeit ehrenamtlicher Organisationen. Ihr Fokus liegt jedoch nicht auf der breiteren Geschichte des Freiwilligensektors oder der Zivilgesellschaft, sondern spezifischer darauf, was sie als NGOs bezeichnen. Damit widmen sie sich einer ausdrücklich politischen Unterkategorie des ehrenamtlichen Sektors, die sich mit der Kampagnen- und Lobbyarbeit befasst. Unvermeidlich führt ein solcher Schwerpunkt zu einem wissenschaftlichen Projekt, dass letztlich Fragen zur Politikgeschichte und nicht mehr so sehr zur Sozialgeschichte beantworten will.

II

Vor dieser politischen Wende hatte Wohltätigkeit seinen vorhersehbaren Platz als ein Thema innerhalb einer weiteren Sozialgeschichte, die sich häufig auf Aspekte der Armut und auf das Verhältnis zwischen Arm und Reich konzentrierte. Obwohl in den klassischen Werken, die sich um eine möglichst vollständige Geschichte britischer Philanthropie bemühten, das Wohltätig Sein meist das Wohltätigkeit Empfangen ausschloss, boten sie doch eine Plattform für Sozialhistoriker, sich eingehender mit diesem Verhältnis zu befassen. ${ }^{18}$ In seinem wegweisenden Outcast London lenkte 
Gareth Stedman Jones unsere Aufmerksamkeit auf die "Deformation des Geschenks": Die physische Trennung der gesellschaftlichen Klassen durch Suburbanisierung bedeute, dass die Beziehungen zwischen ihnen - und damit auch wohltätige Begegnungen - anonymisiert und abstrahiert würden. ${ }^{19}$ Diese Ausrichtung der investigativen Sozialgeschichte zeigt traditionell eine hohe Bereitschaft, anthropologische Ansätze zu Schenkungs- und Tauschverhältnissen aufzunehmen.

Das Konzept, dass Wohltätigkeit den mittleren und oberen Gesellschaftsschichten die „soziale Kontrolle" über ihre Nachbarn aus der Arbeiterklasse - und besonders über die Armen - ermöglichte, war in dieser frühen Phase der Sozialgeschichte der Wohltätigkeit möglicherweise am einflussreichsten. Freilich gab es keine einzige, allgemein akzeptierte Auffassung dessen, was soziale Kontrolle denn sei. Einige Historiker sehen diese als „bewusstes Handeln“ seitens der britischen "herrschenden Schicht", das darauf gezielt habe, "die bestehende soziale Einseitigkeit gesellschaftlicher Beziehungen" aufrechtzuerhalten. ${ }^{20}$ In anderen Fällen erwies es sich als produktiver, sie als stillschweigend und als eng verbunden mit den Austauschbeziehungen von Rechten und Verantwortungen eines jeden Wohlfahrtssystems zu begreifen, dem „ein Code autoritärer Regeln“ zugrunde liegt. ${ }^{21}$ Dieser Ansatz trifft auch auf kritische Stimmen. Ende der 1970er Jahren schrieb Karel Williams, dass „englische Sozialhistoriker sich bemüht haben, eine Beziehung zwischen Sozialgeschichte und Soziologie aufzubauen, in der Art wie neue Wirtschaftshistoriker es für Wirtschaftsgeschichte und der Volkswirtschaftslehre getan haben“.22 Dennoch, so Williams weiter, bleibe dabei nicht nur die Unklarheit bestehen, welches Modell „sozialer Kontrolle“ von der Soziologie hinzugezogen werden sollte, sondern es werde damit auch ein "Taschenspielertrick" verwendet, indem das, was der Theorie nach notwendig sei, die tatsächlichen Gegebenheiten ersetze. ${ }^{23}$

2015 fragte Barry Doyle, ob es im Feld der voluntary action history noch jemanden gebe, der von "sozialer Kontrolle" spreche. ${ }^{24}$ Tatsächlich wurde dieses Modell vielfach beiseitegelegt, da seine Problematik letztlich seinen Wert übersteige. Ein alternatives Deutungsmuster, das inzwischen aber ebenfalls in Ungnade gefallen ist, wird durch die Begriffe Handlungsfähigkeit (agency) und Macht (power) repräsentiert. ${ }^{25}$ Macht wird hier im diskursiven Sinn verstanden, wobei eine Art Skript für die wohltätige Begegnung existiere - und zu dem sich die Handlungsfähigkeit entweder in Einklang oder im Widerstand befinde. Wenn diese Terminologie auch nicht allgemein verwendet wird, sind diese Konzepte doch in historischen Narrativen zur Armut deutlich präsent, worauf noch zurückzukommen sein wird. Während die Idee der Wohltätigkeit als Ritual in älteren Arbeiten zur frühneuzeitlichen Geschichte der Sozialfürsorge als typisch gelten kann, passt sie auch in Studien neueren Datums, die sich eher auf Rhetorik und Strategie karitativer Appelle konzentrieren. ${ }^{26}$ Was sich bei Historikern allgemein wie auch bei Historikern der Wohltätigkeit nach wie vor dauerhafter Beliebtheit erfreut, ist die anthropologisch inspirierte Forschung zur "Gabe“, zum „Gabentausch“ und zur „gift relationship“. 
Es ist angebracht, zunächst einen kurzen Blick auf die verschiedenen Forschungsansätze zu diesen Konzepten zu werfen.

Die in der britischen Sozialwissenschaft wahrscheinlich bekannteste Deutung der "gift relationship" stammt von Richard Titmuss. Er wählte diesen Ausdruck 1970 als Titel für seine einflussreiche Studie zu unterschiedlichen Blutspendesystemen weltweit. Besonders positiv bewertete er das britische System, in dem die demonetarisierte Spende an einen anonymen Rezipienten gerichtet war. Hierdurch wurde eine Situation geschaffen, in welcher das Geschenk durch einen gewissen Altruismus charakterisiert wurde und die gesellschaftliche Solidarität förderte. ${ }^{27}$ Interessanterweise garantierte aus Titmuss' Sicht die Trennung von Spender und Rezipienten den Altruismus, wohingegen genau der gleiche Aspekt in der viktorianischen und edwardischen Philanthropie für Gareth Stedman Jones die „Deformation des Geschenks" verursachte. ${ }^{28}$ Die positivere Einschätzung entstammte der kollektivistischen Auffassung, dass die Gesellschaft aus gegenseitigen Wechselverhältnissen bestehe. Diese wird oftmals mit den Arbeiten von Marcel Mauss zum nicht-monetarischen „Gabentausch" in archaischen Gesellschaften in Verbindung gebracht, ebenso wie mit denjenigen von Claude LéviStrauss zum Gegenseitigkeitsverhältnis in verwandtschaftlichen Beziehungen. ${ }^{29}$

Dies steht aber im Kontrast zu dem Ansatz, das Thema aus einer individualistischen Perspektive anzugehen, der im utilitaristischen Denken wurzelt und davon ausgeht, dass soziale Verhältnisse in erster Linie durch Eigeninteresse motiviert sind. Der vielleicht überzeugteste Vertreter dieser Ansicht in der Geschichte der Wohlfahrt ist Alan Kidd, der auf die Bedeutung von „Gegenseitigkeit“ nicht für die Herstellung einer zusammenhängenden Gemeinschaft, sondern für die Entstehung gesellschaftlicher Unterschiede hinweist:

„Die Abhängigkeit des Empfängers von der Gabe und seine Unfähigkeit in der Folge, sie zu vergelten (beziehungsweise eine Möglichkeit dafür vorauszusehen) kann kaum gesellschaftliche Solidarität schaffen. Vielmehr festigt sie soziale Unterschiede und schürt sogar Verbitterung. Der Empfänger wird materiell und moralisch zum Schuldner des Gebers ... Wir wissen, dass Wohltätigkeit selbst die Macht hat, zu verletzen. ${ }^{\text {"30 }}$

Das Fundament dieses Machtverhältnisses liegt darin, dass Philanthropie vom Spender beziehungsweise vom Freiwilligen definiert wird, der über Zeitpunkt, Ausmaß, Methode und Ziel der Wohltätigkeit entscheidet. Im Gegensatz hierzu hat der passive Rezipient wenig Spielraum für eigenes Handeln. Fernand Braudel hat dies in einem einleuchtenden Satz zusammengefasst: „Wer gibt, dominiert". ${ }^{31}$ Wenngleich Braudels Interesse vorrangig der Dominanz einer Kultur über die andere galt, beobachtete er nebenbei, dass dasselbe auch auf Individuen zutreffe. Obwohl dies eher als 
Binsenweisheit präsentiert wurde, war es doch ein deutlicher Ausdruck eines individualistischen Verständnisses der gift relationship.

Ein solcher Rahmen, um das Verhältnis zwischen Spendern und Rezipienten zu analysieren, wurde durch den Einfluss der politischen Wende auf die Sozialgeschichte verdrängt. Stattdessen verschob sich der Interessensschwerpunkt der Sozialhistoriker zu den Beziehungen zwischen ehrenamtlichen und öffentlichen Anbietern sozialer Dienste, während die Vorstellung der "mixed economy of welfare“ aus der Sozialpolitik übernommen wurde. ${ }^{32}$ Die Erkenntnis, dass es eine Epoche nach dem „klassischen Wohlfahrtsstaat" geben könne, in welcher der öffentliche Sektor eine kleinere Rolle spielen würde, führte Historiker zur Bestätigung der Tatsache, dass „Sozialfürsorge immer gemischt“ gewesen sei. ${ }^{33}$ Seither wurde der konzeptuelle Rahmen der mixed economy of welfare auf die historische Analyse des gesamten Bereichs der sozialen Dienstleistungen angewandt. So haben Anne Digby und David Wright diesen Ansatz bei der Geschichte der Dienste für Erwachsene mit Lernschwierigkeiten verwendet. ${ }^{34}$ Später haben Graham Chester und Pamela Dale die naheliegende Parallele zur gegenwärtigen Politik weitergeführt, indem sie innerhalb der Geschichte der mixed economy in Bezug auf Dienstleistungen für Erwachsene mit Lernschwierigkeiten zwischen „Käufern“ und „Anbietern“ unterscheiden. ${ }^{35}$

Das Verständnis von Vielfalt in der Dienstleistungsbranche aus der Sicht der "mixed economy" ist mittlerweile allgemein Standard geworden, selbst dort, wo der Begriff nicht explizit benutzt wird. Oft werden für ähnliche Forschungsansätze zum Thema alternative Konzepte wie etwa „governance“, Wohlfahrtspluralismus oder Beveridges „moving frontier“ zwischen ehrenamtlicher Arbeit und Staat herangezogen. Richard Trainor und Robert Morris argumentieren, dass das politikwissenschaftliche „Governance“-Konzept nicht nur die städtische Sphäre miteinbeziehe, die von so vielen früheren Studien bereits behandelt wurde, sondern es ebenso erlaube, andere Dienstleister, etwa "die nichtstädtischen Kooperationspartner lokaler Verwaltungsapparate, freiwillige Institutionen" oder "die Organisationen des professionellen und wirtschaftlichen Sektors" zu berücksichtigen. ${ }^{36}$ Welche Terminologie auch immer verwendet wird: Die Konsequenz ist, dass neue und überarbeitete Überblicksdarstellungen zur Geschichte der Sozialfürsorge in Großbritannien nun in der Regel eine Diskussion ehrenamtlicher Armen- und Krankenversorgung mit einschließen. ${ }^{37}$ Dies verschafft der Geschichte der Wohltätigkeit zwar einen sicheren, aber noch immer untergeordneten Platz in der weiteren Geschichte der Wohlfahrt. Ob sie in erster Linie als soziale oder politische Geschichte betrachtet werden soll, richtet sich größtenteils danach, welche größeren Forschungsfragen gestellt werden. Im Fall einiger hervorragender Arbeiten zu wichtigen sozialen Themen wie etwa Pat Thanes und Tanya Evans' Studie zu unverheirateten Müttern, die sie zum hundertjährigen Jubiläum einer ehrenamtlichen Einrichtung verfasst haben, ist die Wohltätigkeitsgeschichte Teil einer größeren politischen Geschichte. ${ }^{38}$ 
Wie oben erwähnt, lässt sich die andere Konsequenz der politischen Wende als wissenschaftliche Vernachlässigung der gesellschaftlichen Bindungen zwischen Arm und Reich beschreiben, die durch karitatives Handeln entstehen können, wohingegen eine Reihe neuer Themen erschlossen wurde: etwa die sozialen Bindungen und Netzwerke, die sich innerhalb bestimmter Schichten und auch zwischen den Mitgliedern freiwilliger Organisationen herausbildeten. Im Gegensatz zum Narrativ des Rückgangs bürgerlichen Engagements, das unter dem Einfluss von Robert Putnams Argumenten zur USA an Stärke gewann, wollen Historiker in jüngster Zeit eher die Bedeutung des Vereinslebens in der britischen Vergangenheit wieder zum Vorschein bringen, etwa auch als Teil der Freizeit-, Bildungs-, Jugendkultur- und Politikgeschichte. ${ }^{39}$ Dies war eine neue Dimension, welche die VAHS ausloten wollte, als sie in den 2000er Jahren eine neue Seminarreihe am University of London's Institute of Historical Research veranstaltete. Gleichzeitig übernahmen Historiker leitende Positionen in der VAHS, nämlich Pat Starkey als Vorsitzender und Georgina Brewis als Schriftführerin. Damit bedeutete jedoch nicht, dass alle anderen Themen ausgeschlossen wurden. Starkeys eigene Forschung zur Bedeutung freiwilliger Organisationen im Bereich der Sozialarbeit ist weniger in der politischen Geschichte freiwilligen Engagements als in der politisierten Sozialgeschichte der Wohlfahrt zu verorten. ${ }^{40}$ Ebenso haben die Arbeiten anderer wichtiger Köpfe in der VAHS vor allem mit der politischen Geschichte von NGOs gemein, wobei auch sie wichtige neue Forschungsstränge in die Sozialgeschichte freiwilliger Organisationen eingebracht haben. Brewis' Arbeit zur Freiwilligenarbeit von Studierenden sowie diejenigen von Caitriona Beaumont zu Frauenorganisationen brachten beide die Erkenntnis voran, dass die Erfahrung von Freiwilligen und Mitgliedern freiwilliger Assoziationen unbedingt mit berücksichtigt werden müssen. Außerdem haben diese Wissenschaftlerinnen herausgearbeitet, über welche Wege diese mit ihrer Freiwilligentätigkeit dazu beigetragen haben, neue politische Gemeinschaften in ihren jeweiligen Wirkungsbereichen zu schaffen. ${ }^{41}$

Im Rahmen eines vielseitigen Forschungsprogramms gibt es mehr Raum für die Geschichte gemeinnütziger Organisationen. Die Bedeutung der Vereinskultur und Zivilgesellschaft für die Entstehung einer bürgerlichen Identität ist schon lange bekannt. ${ }^{42}$ Ein deutlicher Unterschied dieser neuen Vereinsgeschichte war die Hinwendung von den bürgerlichen und karitativen Assoziationen zu neuen Bereichen wie Sport und Freizeit als Formen freiwilliger Tätigkeit. ${ }^{43}$ Eine solche Geschichtsschreibung ermöglichte wertvolle neue Einblicke auch in die Geschichte von Wohltätigkeitsinstitutionen als einer Form der sozialen Organisation, etwa mit Blick auf ihre Fähigkeit, soziales Kapital für ihre Mitglieder und Netzwerke für das Gemeinwesen zu schaffen. Dabei rückte sie allerdings von einer Geschichte der Wohltätigkeitsinstitutionen als einer Form sozialer Interaktion ab. Letzteres Feld ist seit der politischen Wende in der Historiographie zur Wohltätigkeit in der Regel nur noch von verschiedenen kulturgeschichtlichen Blickwinkeln aus untersucht worden. 
Seth Kovens innovative Arbeit untersucht visuelle Repräsentationen armer Kinder und kriegsversehrter Veteranen in dem sozialen und sexuellen Kontext, in dem sie bei Spendenkampagnen dargestellt wurden. ${ }^{44}$ Andere Arbeiten haben die materielle Seite karitativer Dienste analysiert, indem sie die Bemühungen, Arme und Bedürftige mit Kleidung zu versorgen, in den Blick genommen haben, oder haben die universitären Settlements als Orte des Aufeinandertreffens verschiedener Klassen untersucht. ${ }^{45}$ Diese verschiedenen Studien haben neues Licht auf die Geschichte karitativer Begegnungen geworfen, und folgten somit auf gewisse Weise Alan Kidds Aufruf:

„Das Kräftefeld in dem die Philanthropie angesiedelt ist, entspricht einem System materieller und kultureller Beziehungen, deren Analyse ebenso Handlungsfähigkeit wie auch Struktur angemessen berücksichtigen sollte; Aufmerksamkeit gebührt auch der Form ebenso wie dem Inhalt bei der Untersuchung kultureller Artefakte vom Jahresbericht bis zum Wohltätigkeitsbasar. Die schriftliche und symbolische Sprache der Philanthropie wartet darauf, von uns erschlossen zu werden. ${ }^{\prime 46}$

Diese beiden Sprachen haben jedoch noch nicht wirklich miteinander kommuniziert und somit auch nicht kollektiv zu einer erneuerten, neu ausgerichteten soziokulturellen Geschichte der Wohlfahrt beigetragen, welche die ältere Sozialgeschichte ersetzen könnte.

Kovens Arbeit dazu, wie Wohltätigkeitsempfänger wahrgenommen wurden, ist vielleicht am originellsten, aber auch sie fängt nicht die Stimmen der Rezipienten selbst ein. In der Tat ist eine Geschichte der Sozialfürsorge „von unten“, so wertvoll sie auch erscheint, besonders schwierig zu rekonstruieren. Wie Pat Starkey anmerkt, sind die in den 1970ern und 1980ern unternommenen Versuche, den Fürsorgeempfängern eine Stimme zu verleihen, deshalb fehlgeschlagen, weil sie die Dynamik der Machtverhältnisse nicht erfassten, mit der sie es zu tun hatten. ${ }^{47}$ Denn für eine solche Erfahrungsgeschichte müssen wir berücksichtigen, dass das Ungleichgewicht der gift relationship nicht nur die Gabe selbst betrifft, sondern auch die historische Überlieferung zu dieser Gabe. Das historische Quellenmaterial verleiht unvermeidlich der Perspektive derjenigen ein größeres Gewicht, die eine Machtposition innehalten. Ähnliche und vergleichbare Probleme haben in der Medizin- und in der Wohlfahrtsgeschichte Aufmerksamkeit auf sich gezogen. Die Schwierigkeiten dabei sind oft hervorgehoben worden, so auch von John Pemble:

„Viktorianische Armut ist stumm, entweder weil die von ihre Betroffenen [in den Worten des liberalen Politikers Charles Masterman aus dem Jahr 1902] ,großenteils die Ausdrucksfähigkeit fehlte' oder weil sie sich zu sehr schämten, um darüber zu sprechen. Die Zeitgenossen, die über sie schrieben, waren Fremde in ihrer Mitte: Missionare aus dem Bürgertum, Philanthropen und Soziologen ..., die sich als verwegene Entdecker, Wissenschaftler oder als in den Slums 
recherchierende Journalisten darstellten. Sie versuchten, eine Erfahrung zu teilen, zu der sie nie vorzudringen in der Lage waren, weil sie wussten, dass sie daraus jederzeit entfliehen konnten.

Viktorianische Armut wurde belagert von Außenstehenden, die von außen hineinsahen. Deswegen wurde auch etwas dagegen getan, und deswegen hat sie eine Geschichte. Aber es ist eine Geschichte der Erforschung, Quantifizierung, Beratung, Gesetzgebung und Vorstellungskraft. Als menschliche Erfahrungsgeschichte existiert sie nicht. ${ }^{\text {"48 }}$

Eine Geschichte der karitativen Begegnung zu schreiben bedeutet in der Regel, Quellen gegen den Strich zu lesen, die von denjenigen geschaffen wurden, die Almosen gaben und nicht von denjenigen, die sie empfingen. Helen Rogers hat in ihrer Studie zur christlichen Gefängnisbesucherin Sarah Martin die überlieferten Teile des „Liberated Prisoners Book“ untersucht, in dem diese den Kontakt, den sie mit befreiten Gefangenen und deren Familien unterhielt, festhielt. Während etliche Details ihrer Lebenswege auch mittels anderer Quellen belegt werden können, bietet dieses Buch einen einzigartigen Einblick in die Erfahrungen der Gefangenen mit einer karitativen Helferin. So wird eine differenziertere Erzählung der „intimen Beziehungen“ möglich, auf der die Disziplin und Fürsorge der Reformerin beruhte. Dadurch wird nicht nur Widerstand als eine wichtige Form der agency in den Vordergrund gestellt, sondern auch Handlungen, die auf gemeinsamen Werten wie Freundlichkeit oder Verwandtschaft basieren. ${ }^{49}$ Solche Quellen müssen ebenso in ihrem Kontext gelesen werden, wie diejenigen, die direkt von Almosenempfängern verfasst wurden. Virginia Crossmanns kürzlich erschienene Untersuchung der Rhetorik schriftlicher Gesuche um wohltätige Unterstützung in Irland nach der Großen Hungersnot zeigt, dass diese neben Strategien der Respektsbezeugung, ein Verlangen nach finanzieller Unabhängigkeit und emotionale Hilferufe aufweisen - wie sie auch in anderen Arten von Unterstützungsgesuche armer Menschen üblich waren. ${ }^{50}$ Die Tatsache, dass solche Quellen nicht von der Machtdynamik der gift relationship, in deren Rahmen sie entstanden sind, getrennt werden können, heißt nicht, dass sie kein Licht darauf werfen können.

III

Neue Schwerpunkte und bislang wenig beachtete Quellen können historische Einblicke in die Erfahrungen von Almosenempfängern verschaffen. Es gibt jedoch auch einen anderen Ansatz, der auf der Einsicht aufbaut, dass Wohltätigkeit - als einen (meist vermittelten) Transfer von Geld, unbezahlter Arbeit oder anderer Ressourcen - letztlich eine wirtschaftliche Aktivität darstellt. Der typische Fokus auf die sozialen Aspekte karitativen Handelns, unter Vernachlässigung der wirtschaftlichen Aspekte, spiegelt eine breitere Tendenz in der Sozialgeschichte wieder, diese beiden Dimensionen als grundsätzlich voneinander verschieden zu betrachten: als lägen ihnen jeweils andere Funktionslogiken zugrunde mit unterschiedlichen Grundprinzipien, die daher auch eine andere 
Analyse verlangten und schließlich auch unterschiedliche Geschichtsschreibungen, von verschiedenerlei Historikern verfasst. Dies wird zwar nicht bewusst in die Methodologie der Sozialgeschichte eingeflochten, ist aber doch der allgemein angenommene Ausgangspunkt. Diese Annahme ist allerdings nicht gesichert, wie ein Blick auf die Wirtschaftssoziologie zeigt. Hier stellt sie eine von drei verschiedenen Grundhaltungen dar, die das Verständnis modernen wirtschaftlichen Verhaltens prägen. Da uns alle drei auch zu unterschiedlichen Ansätzen zur Geschichte der Wohltätigkeit führen können, lohnt es sich, einen Blick auf sie werfen.

Historiker ebenso wie Anthropologen, Soziologen und Vertreter anderer Disziplinen haben den Akt des Schenkens lange und genau studiert. Dadurch haben sie die unzähligen Bedeutungen und Implikationen des Anbietens, Annehmens und Ablehnens eines Geschenks angemessen erschlossen. Dennoch liegt bis heute keine gleichwertige Geschichte des weitaus häufigeren gesellschaftlichen Akts der Bezahlung vor. Es gibt allerdings eine soziologische Literatur zur gesellschaftlichen Bedeutung des Geldes, die in den 1990er Jahren neu belebt wurde, um einige ihrer Fragen im Kontext des Anstiegs amerikanischer Konsumentenkultur anzusprechen. Damit sollte ein älteres, utilitaristisches Gedankengebäude zu Geld und seiner Bedeutung ersetzt werden. Die Grundannahme des neuen Ansatzes war, dass die komplexen sozialen Beziehungen der vorindustriellen Ära abgeflacht und durch einen einfachen "Cash Nexus" ersetzt worden sind. Die Terminologie stammte wohl von Thomas Carlyle, der in den frühen Jahren der Regentschaft Königin Viktorias bemängelte, in der neuen Wirklichkeit sei „cash-payment the only nexus of man to man!“51 Die hier zum Ausdruck kommende Auffassung, dass Geld zum einzigen Vermittler zwischen Arm und Reich geworden sei, findet sich auch bei Friedrich Engels' Werk Die Lage der arbeitenden Klasse in England und Karl Marx beschreibt sie im Kommunistischen Manifest als Triumph, den das bürgerliche "nackte Interesse“ über die feudalen Beziehungen der Vergangenheit errang. ${ }^{52}$ Ungeachtet der einflussreichen Arbeit Emile Durkheims, die den Wandel als den Ersatz alter Solidaritätsverhältnisse durch neue interpretierte, lebt die Sorge über aufgelöste soziale Bindungen über die Generationen hinweg fort. ${ }^{53} \mathrm{Im}$ Einklang mit den aus der Industrie vernehmbaren Klageliedern hat Anthony Giddens kürzlich dem Geld eine bedeutende Rolle für die Herausbildung der Moderne zugeteilt. Er beschreibt es als eines der „symbolischen Zeichen“, die als „entbettender Mechanismus“ fungieren und soziale Verhältnisse aus ihren regionalen Zusammenhängen ",herausheben“. ${ }^{54}$

Historiker setzen sich nur selten mit diesem speziellen Argument auseinander. Aber die vielfältige Forschung zur gesellschaftlichen Bedeutung des Geldes in der Frühen Neuzeit findet im modernen Zeitalter der Abstraktion keine Entsprechung. ${ }^{55}$ Schulden und Kredit beispielsweise werden im Ausnahmefall als sozial behandelt. ${ }^{56} \mathrm{Implizit}$, indes fast nie explizit, wird damit vorausgesetzt, dass modernes Wirtschaftsverhalten in der Regel nicht gesellschaftlich bedingt ist, sondern in einem rein 
wirtschaftlichen Zusammenhang funktionsfähig und erklärbar ist. Die Voraussetzung dafür, dass solche geldbezogenen Konzepte, wie sie hinter der Trennung zwischen der Sozialgeschichte und der Wirtschaftsgeschichte des Alltags stehen, als entbettend betrachtet werden, ist die Annahme, Geld selbst sei wertneutral.

Dieses Denken hat eine lange Tradition. Seinen ersten Ausdruck fand es um 1900 in Georg Simmels Die Philosophie des Geldes, in der dieser Geld als "das Gelten der Dinge ohne die Dinge selbst" definierte beziehungsweise als „dasjenige konkrete Mittel, das sich mit dem abstrakten Begriffe desselben ohne Abzug deckt". So verstanden war Geld gleichermaßen Nivellierer und Befreier. Beides könne es sein, gerade weil es „das Mittel schlechthin“ sei. ${ }^{57}$ Auch Max Webers soziologische Auslegung des Bezahlens als eine wirtschaftliche Transaktion fasste diese nicht als Kennzeichen der Industrialisierung auf, sondern als universelle Form sozialer Interaktion. Geld war für geschichtliche Entwicklungen und Veränderungen unentbehrlich, aber entstand nicht aus ihnen. Es konnte also ein Mittel sein, das genutzt wurde, um dem kalvinistischen "Ruf" entsprechend zu handeln, um den Feudalismus durch Individualismus zu ersetzen oder aber um die moderne Maschinerie der Bürokratie herbeiführen. ${ }^{58}$ Eine ähnliche wertneutrale Sicht des Geldes findet sich in der Soziologie der 1950er Jahre bei Talcott Parsons, für den Geld ein Symbol war, ein neutrales Medium der Interaktion, das maßgeblich nichts dazu tat, um die Realwirtschaft oder auch die sozialen Beziehungen zu verändern, in deren Rahmen es eingesetzt wurde. In Parsons' Arbeiten war Geld eine Sprache, die zwar Wert übermitteln konnte, aber zu Werten schwieg. ${ }^{59}$

Diese utilitaristische und beklagende Sicht des Geldes floss unbewusst in die theoretischen Ansätze zur Geschichte der Wohlfahrt ein, die dem Deutungsansatz der „Sozialkontrolle" folgen und somit ungeachtet des jeweiligen moralischen, kulturellen oder sozialen Kontextes stets ökonomische Macht am Werke sehen. Andere Perspektiven vermeiden die Theorie grundsätzlich. Studien von Brian Harrison und Frank Prochaska z.B. weisen auf Formen der Wohltätigkeit hin, die, wie etwa die gegenseitige Unterstützung der Arbeiter, innerhalb einer Klasse geleistet wird. ${ }^{60}$ Wie Colin Jones in den 1990er Jahren schrieb, „verkompliziert dies auf furchtbare Weise die Zweierbeziehung von Geber und Empfänger, auf der ein Großteil der Forschung zur Geschichte der Wohltätigkeit basiert" ${ }^{61}$ Solch verkomplizierte Sichtweisen wurden typischerweise auf empirischem Wege präsentiert, die - wenn überhaupt - nur ansatzweise einen alternativen theoretischen Rahmen boten, um von diese Beispielen aus ein weiter reichendes Argument zu entwickeln und die Beteiligung des Arbeiterstandes an freiwilliger Tätigkeit als Illustration eines gemeinsamen Anliegens jenseits aller Klassenunterschiede zu deuten. Demgegenüber ist im Bereich der Neuen Wirtschaftssoziologie ein theoretischer Rahmen entwickelt worden, an dem wir unsere Analyse ausrichten können. 
Die Neue Wirtschaftssoziologie ist ein vielseitiges Gebiet, und einige Forschungsbereiche sind hier von größerem Nutzen als andere. Es gibt etwa ein sogenanntes „Erweiterungs-“ Projekt, das untersuchen soll, inwiefern wirtschaftliche Logiken in Bereichen funktionieren, die nicht traditionell als wirtschaftlich angesehen werden. Hiermit wird ein Pfad eingeschlagen, der mit demjenigen einher läuft, den wir gerade verlassen wollen. ${ }^{62}$ Es wird hier von einer enggefassten Logik eines wohlkalkulierten Eigeninteresses als Triebkraft im wirtschaftlichen Verhalten ausgegangen, mit der sich aber das Phänomen der Wohltätigkeit kaum erklären lässt. Eine Lösung wäre hier, sich auf die greifbaren Resultate philanthropischer Tätigkeit zu konzentrieren, zum Beispiel ein „bestimmte Art des persönlichen Status", die durch karitative Tätigkeit gewährt wird, etwa indem Parlamentskandidaten die von ihnen erwarteten lokalen Führungsqualitäten zugesprochen werden. ${ }^{63}$ Dies führt uns jedoch schon in einen weiteren sozialen Kontext, zum Kennzeichen eines separaten Projekts in der Neuen Wirtschaftssoziologie. Es handelt sich um eines von zwei parallelen Projekten, die beide ihren Ausgangspunkt in der Ablehnung des alten, problematischen Verständnisses davon sehen, wie Geld sich als soziale Technologie auf Wirtschaftsverhalten auswirkt - aber jeweils auf andere Weise. Das eine will ökonomisches Verhalten kontextualisieren, das andere will es neu definieren.

Versuche, ökonomische Tätigkeit zu kontextualisieren, werden mit der Forschung Mark Granovetters assoziiert, dessen 1985 erschienener Artikel zum „Problem der Eingebundenheit“ als Gründungstext der neuen Wirtschaftssoziologie betrachtet werden kann. Granovetter lehnte das, was er als „die Sichtweise der Mehrheit der Soziologen, Anthropologen, Politologen und Historiker" bezeichnete, rundweg ab, dass nämlich die Modernisierung das ökonomische Verhalten von sozialen Beziehungen löste. Er wies jedoch ebenso die utilitaristische, (neo)klassische ökonomische Perspektive zurück, die sich auf universell unabhängiges (wie auch rationales und eigennutzorientiertes) Wirtschaftsverhalten stützte. Beide Ansätze wurden von Granovetter kritisiert, weil sie Menschen als atomisierte Akteure ansahen, selbst wenn diese nach internalisierten Verhaltensregeln handelten. Stattdessen forderte er, in weitaus größerem Maße als bisher, den unmittelbaren sozialen Kontext hinzuzuziehen. ${ }^{64}$ Während Karl Polanyi davon ausging, dass dem „Marktmuster“ der kapitalistischen Wirtschaft gemäß, „soziale Verhältnisse in das Wirtschaftssystem eingebettet sind“, vertrat Granovetter das Gegenteil. ${ }^{65} \mathrm{Im}$ Laufe der vergangenen drei Jahrzehnte entwarf er einen theoretischen Rahmen, um Wirtschaftsverhalten zu analysieren. In diesem Rahmen wird davon ausgegangen, dass ökonomisches Handeln von anderen menschlichen Verhaltensweisen nicht zu trennen ist, und dass es durch dieselben sozialen, emotionalen, kulturellen, legalen, religiösen und anderen Motiven und Einschränkungen bestimmt wird. ${ }^{66}$ 
Viviana Zelizer entwickelte eine andere Herangehensweise. Sie lehnt utilitaristische Betrachtungsweisen des Geldes ebenso ab, ihre Alternative ist jedoch radikaler als die Granovetters. Während er darauf besteht, dass das Ökonomische nicht ohne seinen gesellschaftlichen Kontext verstanden werden kann, kann ihr Ansatz im Wesentlichen als die These beschrieben werden, dass das Ökonomische selbst gesellschaftlich ist. Daraus folgt, dass ihre Arbeit nicht die Abweichungen eines vorhersehbaren Wirtschaftsverhaltens zu erklären sucht, sondern dieses auf einer Ebene mit jedem anderen Sozialverhalten auch behandelt. Sie richtet ihre Aufmerksamkeit auf die Bedeutung interpersoneller Beziehungen für das ökonomische Verhalten in Bereichen, die sonst nicht als ökonomisch betrachtet werden. So untersucht sie, wie Lebensversicherungen gesellschaftliche Anerkennung gewannen, oder wie wir das Leben eines Kindes nach finanziellem Wert bemessen. ${ }^{67}$ Des Weiteren argumentiert sie, dass die Rituale, die sich zum Geldgebrauch entwickelt haben, in keinerlei Weise einen entmenschlichten Fluchtweg aus der gesellschaftlichen Konvention darstellen, sondern vielmehr soziale und kulturelle Werte und Sinnstiftungen vermitteln können:

„Von den austauschbaren, unpersönlichen Eigenschaften des Geldes beeindruckt, haben die klassischen Theoretiker stets seine instrumentale Rationalität und seine scheinbar unbegrenzte Fähigkeit betont, Produkte, Beziehungen und manchmal sogar Emotionen in ein abstraktes, objektives Zahlenverhältnis zu verwandeln. Aber Geld ist weder kulturell neutral noch sozial anonym. Es kann sehr wohl Werte „korrumpieren“ und soziale Bindungen in Zahlen verwandeln, aber Werte und Beziehungen verwandeln Geld im Gegenzug ihrerseits, indem sie ihm Bedeutung und soziale Strukturen verleihen. ${ }^{\circ 68}$

Dies sind jedoch keine Theorien und Studien, mit denen viele Sozialhistoriker vertraut sind, selbst wenn sie das eine oder andere Prinzip daraus als gesunden Menschenverstand widergeben.

Um zu illustrieren, mit welchem Gewinn sich geschichtliche Studien innerhalb dieses theoretischen Terrains verorten können, wenden wir uns der Patientenzahlungen in den voluntary hospitals im Großbritannien des frühen 20. Jahrhunderts zu. Dies waren karitative Einrichtungen, die oftmals bis ins frühe 18. Jahrhundert zurückreichten. Gegründet wurden sie durch wohltätige Assoziationen und durch das Engagement der mittleren und oberen Gesellschaftsschichten, um kranken Bedürftigen medizinische Pflege zukommen zu lassen und diese im Laufe der nächsten 200 Jahre zunehmend auf die Basis wissenschaftlicher Erkenntnisse zu stellen. ${ }^{69}$ Diese Krankenhäuser entwickelten sich allmählich zu Spitzeninstitutionen klinischer Medizin, die bewusst mit den führenden Krankenhäusern in Deutschland und den USA einherschritten. Bald assoziierte man sie mit der prestigereicheren Behandlung akuter Fälle, während die Dienstleistungen öffentlicher Krankenhäuser, die dem 
Armengesetz unterstanden und in erster Linie Infektionskrankheiten behandelten, expandierten, um den Bedürfnissen der Mehrzahl der Patienten gerecht zu werden. ${ }^{70}$

Nur drei Jahrzehnte lang war es in diesem System die Regel, dass Patienten dem Krankenhaus, in dem sie behandelt wurden, etwas zahlen mussten: zwischen dem Ende des Ersten Weltkriegs und 1948, als alle Krankenhäuser in Großbritannien in den staatlichem Gesundheitsdienst integriert wurden. Vor dieser Zeit wurden Krankenhausleistungen für diejenigen, die es sich nicht leisten konnten, einen Arzt zu bezahlen, entweder von einer wohltätigen Organisation oder vom Staat übernommen. Schon im frühen 20. Jahrhundert begannen die Auswahlkriterien hierfür aufzuweichen, nicht zuletzt weil medizintechnische Entwicklungen für die modernsten Behandlungen zunehmend Krankenhausaufenthalte voraussetzten. $\mathrm{Zu}$ dieser Zeit wuchs der Bedarf nach Krankenhausbehandlungen, und zwar vor dem Hintergrund eines immer schwierigeren Wirtschaftsklimas, zu dem sich finanzielle Probleme gesellten, weil die staatliche Erstattung für den Beitrag der voluntary hospitals für die Kriegsanstrengungen geringer ausfiel als erwartet. All diese Faktoren führten zu einer Situation, in der die Krankenhäuser zwar immer mehr tun konnten, sich aber immer weniger zu leisten vermochten. Deshalb bemühten sie sich aktiv um neue Einnahmequellen, wozu auch die Patienten selbst gehörten. ${ }^{71}$

Wenngleich die karitativen Krankenhäuser allesamt unabhängig waren, führten sie im Laufe der 1920er Jahre rasch ein verblüffend einheitliches System ein, in dem es drei verschiedene Zahlungsmöglichkeiten gab. Das häufigste Bild zum Wort „Bezahlung“ ist das einer kommerziellen Gebühr, die mittelständische Patienten für ein privates (meist Einbett-) Zimmer entrichten, wodurch sowohl das Krankenhaus als auch der behandelnde Arzt oder Chirurg mit dem Patienten Profit machen konnten. Hierauf bezog sich auch der Historiker John Pickstone, als er „die Invasion kommerzieller Medizin in den Bereich des Krankenhauses" beschrieb. ${ }^{72}$ Für die USA, wo die Mehrzahl der Krankenhauszimmer schon zur Zeit des Zweiten Weltkriegs von Privatpatienten belegt wurde, wäre diese Charakterisierung zutreffend gewesen. In Großbritannien sah es jedoch anders aus. ${ }^{73}$ Selbst zu ihrer Höchstzeit belegten Privatpatienten dort in den voluntary hospitals nie mehr als $9 \%$ der Betten, beziehungsweise, nimmt man die voluntary hospitals und öffentlichen Krankenhäusern zusammen, nur zwischen 3 und $4 \% .{ }^{74}$ Dies bedeutete zwar, dass Privatzimmer im 20. Jahrhundert ein Merkmal des Krankenhauses geworden waren. Aber es gilt zu bedenken, dass es Einkommensgrenzen gab, die sicherstellten, dass diese Regelung nur für die 10-15\% der Bevölkerung gültig war, deren Einkommen zu hoch lag. Auf den gewöhnlichen Krankenstationen, die keine Privatpatienten aufnahmen, gab es zwei andere Zahlungsmodalitäten. 
Die meiste historiographische Aufmerksamkeit ist dem beitragsgebundenen Krankenhaussystem zuteil geworden. Dieses geht zum einen auf Spendensammlungen zurück, zum anderen auf gegenseitige Hilfeleistungen. Mitglieder, die sich an einem solchen beitragsgebundenen System beteiligten, zahlten jede Woche einige geringe Summe ihres Gehalts ein, im Gegenzug erfolgte im Falle einer Einweisung eine Zahlung an das Krankenhaus. Zu den Auswirkungen dieses beitragsgebundenen Systems auf wohltätiges Handeln in Krankenhäusern sind einige kühne Behauptungen aufgestellt worden. So folgerte Steven Cherry, dass dieses „versicherungsähnliche“ System einen radikalen Bruch mit den respektvoll-unterwürfigen philanthropischen Traditionen darstellte. ${ }^{75}$ Martin Gorsky und John Mohan hingegen haben behauptet, dass beitragsgebundenen Systeme "von ihren Einzahlern vom Charakter her wie eine Versicherung" wahrgenommen wurden und dass sie durch ihre Zahlungen im Krankheitsfall ein "moralisches Recht“ auf eine Krankenhausbehandlung erworben hätten. ${ }^{76}$ Beide Deutungen gehen im Grunde von einer Neuausrichtung aus, sei es von medizinischer Fürsorge hin zu medizinischem Konsumverhalten, sei es von der Philanthropie des viktorianischen Liberalismus hin zur freiwilligen Gesundheitsfürsorge eines anbrechenden sozialdemokratischen Zeitalters.

Um indes das Verhältnis von Wandel und Kontinuität zu begreifen, sollte man das beitragsgebundene System mit einer weiteren Zahlungsmöglichkeit vergleichen - nicht zuletzt, weil Ersteres allenfalls etwa ein Viertel der Bevölkerung abdeckte. Dies ist beeindruckend, aber die Mehrheit der ins Krankenhaus eingewiesenen Patienten konnte aber nicht auf diese finanzielle Absicherung zugreifen, sondern musste stattdessen einer Lady Almoner Rede und Antwort stehen. Diese tauchten erstmals in den 1890er Jahren in Londoner Krankenhäusern auf, wurden während der 1920er und 1930er im ganzen Land zur Regel, während sich gleichzeitig allmählich die Bezeichnung „medical social worker“ durchsetzte. Diese Fürsorgerin übte im Krankenhaus das aus, was wir heute als Sozialarbeit oder arbeitsmedizinische Beratung bezeichnen. Sie wurde explizit dafür eingestellt, für Patienten das Zahlungsverfahren zu regeln. Nachdem die Entscheidung getroffen worden war, den Patienten einzuweisen, wurde ein Gespräch geführt, um die Bedingungen des Krankenhausaufenthalts festzulegen. Das konnte heißen, dass von einem Patienten ein Beitrag in Höhe eines Schillings pro Woche verlangt wurde. Dies war keine geringe Summe, belief sich aber nur auf ungefähr ein Drittel der Kosten, die dem Krankenhaus pro Patient entstanden. ${ }^{77}$ Üblicher war es, dass Patienten eine niedrigere Gebühr entrichteten, während der kleinste, aber immer noch bedeutende Anteil der Fälle wurde sogar unentgeltlich behandelt: Dies betraf nicht selten immerhin ein Drittel oder gelegentlich sogar die Hälfte der Patienten. ${ }^{78}$

Wenn alle drei Zahlungsmöglichkeiten in Betracht gezogen werden, wirkt die Veränderung, welche das beitragsgebundene System mit sich brachte, weniger dramatisch. Gewiss spielt in gewissem Maße ein wirtschaftliches Eigeninteresse eine Rolle, wenn die Patienten die Kontrolle über die Bezahlung 
selbst übernahmen und die Kosten, die sonst möglicherweise als große Gesamtsumme zu zahlen wären, auf Raten verteilen konnten. Dies ist jedoch nicht mit dem Kauf einer Krankenhausbehandlung gleichzusetzen. Zahlungsunfähigkeit war kein Hindernis für die Einweisung eines Patienten, und das Personal bot seine medizinischen Dienste nach wie vor auf Honorarbasis an. Berücksichtigt man dies, werden Kontinuitätslinien sehr viel deutlicher als in früheren Studien. Und dieses Ergebnis wird nicht nur bestätigt, indem Zahlungsweisen im größeren Zusammenhang betrachtet werden, sondern auch indem ein anderer Blickwinkel auf das Bezahlen als Wirtschaftsverhalten gewählt wird. Üblicherweise wurde dieses eindeutig als wirtschaftliche Tätigkeit angesehen, die in einem sozialen Zusammenhang stattfindet. ${ }^{79}$ Daher werden Patientenzahlungen unter einem ökonomischen Vorzeichen gedeutet. Selbst wenn die gesellschaftlichen Folgen in Betracht gezogen werden, basiert dies nach wie vor auf utilitaristischen Erwartungen wirtschaftlichen Verhaltens. Alternativ können wir Bezahlung aber auch als einen Mechanismus sozialer Interaktion begreifen, die sich nur als wirtschaftliche maskiert. So müsste man Patientenzahlungen unter sozialem Vorzeichen betrachten, während rein finanzielle Überlegungen nur als ein Faktor unter anderen zu verstehen wäre, die das ökonomische Verhalten lenken. Solche finanziellen Überlegungen mögen für den Systementwurf von Bedeutung gewesen sein, können aber in der Praxis leicht durch Klasseninteressen oder zivilgesellschaftliche Ziele außer Kraft gesetzt werden.

In der Konsequenz dieser anderen theoretischen Herangehensweise wird die Funktion des Geldes nicht notwendigerweise als eindeutig wirtschaftlich gedeutet. Tatsächlich gibt es in britischen voluntary hospitals eine Vielzahl sozialer Funktionen des Bezahlens, die mit den Traditionen medizinischer Wohltätigkeit nicht unvereinbar sind. Es ist unerlässlich, diese zu verstehen, nicht nur, um die Ziele derjenigen nachzuvollziehen, die für das System verantwortlich waren, sondern auch, um die soziale Landschaft medizinischer Wohlfahrt auszuloten, in der Patienten als Empfänger ihre eigenen Entscheidungen treffen dürfen. Zwei dieser Funktionen sind besonders relevant, da sie die klassengebundene Natur und das sozio-moralische Programm der Krankenhäuser als karitative Einrichtungen widerspiegeln.

Die erste soziale Funktion der Bezahlung geht dahin, dass die Verwaltung dieser Zahlungssysteme als Mechanismus dafür diente, Patienten aus der Arbeiterschicht und aus dem Mittelstand zu trennen. Beide erhielten Zugang zu medizinischen Leistungen, aber unter jeweils unterschiedlichen Bedingungen und an unterschiedlichen Orten. Die halbherzige Expansion kommerzieller Dienste für bürgerliche Patienten und die umfangreichen Ausnahmen und Rabatte für Patienten aus der Arbeiterschicht scheinen nicht darauf hinzudeuten, dass es das Ziel der Bezahlung war, dem Profitstreben des Krankenhauses Genüge zu leisten. Vielmehr wurde die Funktion als Einnahmequelle mit derjenigen verbunden, im Aufstieg eines universellen Krankenhaussystems, das allen offenstand, 
zu besänftigen und zu vermitteln. Anstatt dass Geld dazu zu verwendet wurde, sich aus sozialen Konventionen herauszukaufen, diente es dazu, die universelle Gesundheitsversorgung den fest verwurzelten Klassenunterschieden und -hierarchien der Zeit anzupassen. Die Bezahlung bestätigte die traditionellen Werte der Klassengesellschaft. Im Fall der Entbindungskliniken wurden durch die Unterscheidung zwischen verheirateten und unverheirateten Frauen in gleichem Maße wie die Klassenschranken auch moralische Werte aufrechterhalten. ${ }^{80}$ Wirtschaftsverhalten wurde mitnichten aus dem sozialen Kontext herausgehoben. Stattdessen war die Vergütung ein Mittel, um soziale Hierarchien in die voluntary hospitals hineinzubringen. ${ }^{81}$

Die zweite soziale Funktion lag darin, ein neues Ritual für die Einhaltung eines neuen Verhaltenscodexes zu bieten. Seit ihrer Gründung im 18. Jahrhundert hatten voluntary hospitals von ihren Patienten den umfassenden Verhaltensregeln der Institute entsprechend Gehorsam und Unterordnung erwartet. Hierzu gehörte Nüchternheit, das Verrichten von Hausarbeit, und die Bereitschaft, sich nicht nur der medizinischen Behandlung, sondern auch der religiösen Einkehr zu unterziehen. Solche Regeln können als soziomoralischer Codex beschrieben werden. Er wurde durch ein neues Verhaltensmodell ersetzt, mit dem Empfänger der von den Krankenhäusern angebotenen medizinischen Wohltätigkeit ihre Würdigkeit unter Beweis stellten: Dieses bestand in der Zahlung des angemessenen finanziellen Beitrags. Außerdem war es wichtig, das Urteil darüber, was als angemessen galt, zu akzeptieren. Somit war die Bereitschaft, für die Behandlung zu bezahlen, wichtiger als das Bezahlen selbst. Auch hier zeigt sich, dass die Vergütung nicht nur zum Einkommen des Krankenhauses beitrug, sondern dass sie auch dazu diente, seinen guten, bürgerlichen Charakter unter Beweis zu stellen, der von der Fürsorgerin als anständiger Repräsentantin der Mittelschicht kontrolliert wurde. Obwohl dieser Mechanismus, um sich Zugang zu medizinischer Pflege zu verschaffen, zunächst neu und konsumgeprägt erscheinen mag, ist er dem alten System, das den Krankenhäusern bei ihrer Gründung zu Grunde lag, doch sehr ähnlich. So gab es ein Empfehlungssystem für Subskribenten, in dem wohltätige Subskribenten eine gewisse Anzahl Zulassungsscheine für das Krankenhaus erhielten. Diese konnten sie an Personen verteilen, die sie für würdige Empfänger hielten. ${ }^{82}$ Der bürgerliche Torwächter der medizinischen Wohltätigkeit mag sich im Laufe der Zeit professionalisiert haben und zum Teil des Krankenhauses selbst geworden sein, doch das Ritual blieb im Wesentlichen dasselbe. ${ }^{83}$

Dies illustriert, dass das Bezahlen eine Vielzahl sozialer Bedeutungen haben kann und dass soziale Funktionen selbst bei scheinbar eindeutig wirtschaftlichen Transaktionen eine wichtige Rolle spielen können. Für unser Verständnis der Geschichte der Wohlfahrt heißt das, dass wir nicht lediglich eine wirtschaftliche Aktivität betrachten, die in einem von ihr loszulösenden gesellschaftlichen Kontext und zu einem von ihr unabhängigen Zweck zur Anwendung kommt. Für Sozialhistoriker mag es 
selbstverständlich erscheinen, sich mit den tieferen Zusammenhängen, in die wirtschaftliche einschließlich karitative - Verhaltensweisen eingebettet sind, auseinanderzusetzen. Wir müssen jedoch ebenso die sozialen und kulturellen Bedeutungen und Funktionen, die diese in sich tragen, berücksichtigen. Dies geht über das hinaus, was Alan Kidd beobachtete:

„Utilitaristische Konzepte des ,eigennutzorientierten Individuums' haben nur begrenzten Wert für das Verständnis von sozialem (und tatsächlich auch von wirtschaftlichem) Verhalten. Entscheidungen werden in einem sozialen, politischen, kulturellen und ethischen Kontext getroffen. Selbst dem individualistischen Kalkül der Austauschtheorie folgend kann freiwilliges kooperatives Verhalten, wie es Wohltätigkeit ist, nicht als ein Mechanismus des Eigeninteresses rationalisiert werden, in den keinerlei internalisierte soziale Ethik, die zu freiwilligem Handeln antreibt, hineinspielt. ${ }^{184}$

Wohltätigkeit ist demnach weder eine wirtschaftliche Verhaltensform, die in ihrer sozialen Kontextualisierung erläutert werden muss; noch ist sie eine Form des Sozialverhaltens, das eine ihr fremde wirtschaftliche Logik übernimmt. Vielmehr sind die beiden weder getrennt noch unterschiedlich. Dieser theoretische Ausgangspunkt führt uns zu einem ganz anderen Fragenbündel, das die soziale Funktion und Bedeutung ökonomischen Verhaltens in den Blick nimmt, und nicht dessen gesellschaftliche Erklärung. Dies kann bei der Frage, wie Wohltätigkeit als vermittelte Begegnung von Spender und Empfänger historisch funktioniert hat, zu einer Neuausrichtung des Fokus führen.

IV

Die Geschichte ehrenamtlichen Engagements hat sich von ihrem ehemaligen Schwerpunkt, der Philanthropie, wegbewegt. Ihr Fokus richtet sich nun vielmehr auf die Frage, was es bedeutete, arm zu sein. Sämtliche neuen Entwicklungen sind willkommen, weil sie unser Verständnis der historischen Rolle und Position freiwilligen Engagements im modernen Großbritannien bereichern. Zusammen haben sie unsere Perspektive austariert, indem sie die Stellung des Rezipienten innerhalb der Wohltätigkeitsgeschichte abschwächen. Diese neuen Schwerpunktbereiche sind gewiss aufregend. Dennoch gibt es daneben auch produktive neue Herangehensweisen, mittels derer wir versuchen können, einige der alten „sozialgeschichtlichen“ Fragen anzusprechen. Hier kann die karitative Begegnung Einblicke in die moderne Klassengesellschaft bieten. Die Arbeiten verschiedener Forscher haben gezeigt, dass im Rahmen der neuen politischen und Verbandsgeschichten Spielraum vorhanden ist, um den Fokus zu verschieben, etwa indem die Erfahrungen von Freiwilligen ins Narrativ mit einbezogen werden. Eine Geschichte der Wohlfahrt jedoch, die den Empfängern keinen Platz lässt, weist an sich schon einen fundamentalen Fehler auf. 
Eine erneuerte Sozialgeschichte der Wohltätigkeit sollte keine Rückkehr zu oder Erweiterung der alten Sozialgeschichten der Philanthropie darstellen. Vielmehr sollte sie neue Ansätze und Konzepte mit an Bord nehmen, und zwar auch aus anderen Disziplinen. Wo politische und Kulturgeschichten das Forschungsfeld belebt haben, war die Folge oft, dass sich ihre Einsichten zerstreuten, anstatt bewusst zu einer zusammenhängenden Geschichte der Wohlfahrt beizutragen. Beim Schreiben einer solchen erneuerten Sozialgeschichte können wir das Thema der Gegenseitigkeit und der gift relationship aufnehmen - nicht nur aus der Perspektive der Anthropologie heraus, sondern auch aus derjenigen der Neuen Wirtschaftssoziologie. Beide Perspektiven erinnern uns daran, dass wir es in erster Linie mit einer Geschichte des Gebens und Empfangens zu tun haben, beziehungsweise des Verhältnisses zwischen denjenigen, die geben, und denjenigen, die empfangen. Die Neue Wirtschaftssoziologie bietet uns eine Vorlage, einen Werkzeugkasten und die Begrifflichkeiten, mit denen wir beginnen können, einige unserer Einsichten zu einer Erfahrungsgeschichte der karitativen Begegnung zusammenzufügen. Damit können wir anregende neue Dimensionen der Geschichte des ehrenamtlichen Engagements innerhalb und außerhalb Großbritanniens eröffnen.

\footnotetext{
${ }^{1}$ Einen guten Überblick über die Beziehung zwischen der Thatcherregierung und dem Freiwilligensektor liefern Nicholas Deakin, The perils of partnership: the voluntary sector and the state, 1945-1992, in: Justin Davis Smith/Colin Rochester/Rodney Hedley (Eds.), An Introduction to the Voluntary Sector. London 1995, 55-62; sowie Jeremy Kendall/Martin Knapp, The Voluntary Sector in the UK. Manchester 1996, 133-164. Für eine Darstellung, die sich auf den Einfluss des Denkens der Neuen Rechten konzentriert, vgl. Geoffrey Finlayson, Citizen, State, and Social Welfare in Britain 1830-1990. Oxford 1994, 357-400.

2 Zur Voluntary Services Unit, vgl. Matthew Hilton/Nick Crowson/Jean-François Mahout/James McKay, A Historical Guide to NGOs in Britain: Charities, Civil Society and the Voluntary Sector since 1945. Basingstoke 2012, 303-307. Zur Regierung Blair vgl. Jane Lewis, New Labour's Approach to the Voluntary Sector: Independence and the Meaning of Partnership, in: Social Policy and Society 4, 2005, Nr. 2, 121-131.

${ }^{3}$ Finlayson (wie Anm. 1), 358.

${ }^{4}$ Margaret Thatcher, Rede vor der Zürcher Volkswirtschaftlichen Gesellschaft ("The New Renaissance”), 14. März 1977. Online verfügbar unter: http://www.margaretthatcher.org/document/103336.

${ }^{5}$ Margaret Thatcher, Rede vor der General Assembly of the Church of Scotland, 21. März 1988. Online verfügbar unter: https://www.margaretthatcher.org/document/107246.

${ }^{6}$ Margaret Thatcher, Rede bei der Women's Royal Voluntary Service National Conference ("Facing the new challenge"), 19. Januar 1981. Online verfügbar unter: http://www.margaretthatcher.org/document/104551.

${ }^{7}$ Gordon Brown, Back to Begging, in: The Times, 3. Mai 1988.

${ }^{8}$ Die wirtschaftlichen Reformen der Thatcher-Regierungen lösten auch freiwillige Hilfsaktionen in der Form gegenseitiger Unterstützungsnetzwerke für streikende Gemeinden aus. Ob dies jedoch zu einer besseren
} 
Koordinierung von Gemeindetätigkeiten führte und ob diese zunehmende Bereitschaft sich als dauerhafte Einstellung niederschlug, ist schwer zu sagen.

${ }^{9}$ Finlayson (wie Anm. 1), 374-375.

${ }^{10}$ Ebd., 234-235; Nicholas Deakin/Justin Davis Smith, Labour, charity and voluntary action: the myth of hostility, in: Matthew Hilton/James McKay (Eds.), The Ages of Voluntarism: How we got to the Big Society. Oxford 2011, 77,80 .

${ }^{11}$ Matthew Hilton/James McKay, The Ages of Voluntarism: An Introduction, in: ebd., 22; Matthew Hilton/James McKay/Nicholas Crowson/Jean-François Mouhot (Eds.), The politics of expertise: How NGOs shaped modern Britain, Oxford 2013), 54-79.

12 Finlayson, (wie Anm. 1), 304.

${ }^{13} \mathrm{Zu}$ den Auswirkungen auf den freiwilligen Sektor, vgl. Colin Rochester, Rediscovering Voluntary Action: The Beat of a Different Drum. Basingstoke 2013, 85-98.

${ }^{14}$ Deakin (wie Anm. 1), 59-61.

${ }^{15}$ William Beveridge, Voluntary Action: A Report on the Methods of Social Advance. London 1948.

${ }^{16}$ Seine wichtigsten Werke in diesem Bereich sind: Frank Prochaska, Women and Philanthropy in NineteenthCentury England. Oxford 1980; ders., The Voluntary Impulse: Philanthropy in Modern Britain. London 1988; ders., Christianity and Social Service in Modern Britain: The Disinherited Spirit, Oxford 2006. Seine am stärksten in die Tiefe gehende Arbeit für einen Think-Tank ist ders., Schools of Citizenship: Charity and Civic Virtue. London 2002.

${ }^{17}$ Frank Prochaska, Christianity and Social Service in Modern Britain: The Disinherited Spirit. Oxford 2006, viii.

${ }^{18}$ Wilbur Kitchener Jordan, The Charities of London: The Aspirations and Achievements of Urban Society. London 2014 [reprint, Erstveröffentlichung 1960]; David Owen, English Philanthropy 1660-1960. Cambridge, MA, 1964.

${ }^{19}$ Gareth Steadman Jones, Outcast London: A Study in the Relationship Between Classes in Victorian Society. London 2013 [Erstveröffentlichung Oxford 1971], 241-261.

${ }^{20}$ Roy Hay, Employers' Attitudes to Social Policy and the Concept of Social Control, 1900-1920, in: Pat Thane (Ed.), The Origins of British Social Policy. London 1978, 108-109.

${ }^{21}$ John Brown, Social Control and the Modernisation of Social Policy 1890-1929, in: Thane (wie Anm. 20), 126.

${ }^{22}$ Karel Williams, From Pauperism to Poverty. London 1979, 136.

${ }^{23}$ Ebd., 136-139.

${ }^{24}$ Barry Doyle, Voluntary Action History Society $5^{\text {th }}$ international research conference, University of Huddersfield, 10. Juli 2013.

${ }^{25}$ Wenn die Sprache der Macht (power) in der neueren Wohlfahrtsgeschichte verwendet wird, bezieht sich dies meistens auf Lobbyarbeit wohltätiger Organisationen bei politischen Entscheidungsträgern. Vgl. Hilton/McKay/ Crowson/Mouhot (wie Anm. 11).

${ }^{26}$ Zu den älteren Arbeiten, vgl. Roy Porter, The gift relation: philanthropy and provincial hospitals in eighteenthcentury England, in: Lindsay Granshaw/Roy Porter (Eds.), The Hospital in History. London 1989; Mary Fissell, Patients, Power and the Poor in Eighteenth-Century Bristol. Cambridge 1991.

${ }^{27}$ Richard Titmuss, The Gift Relationship: From Human Blood to Social Policy. London 1970. 
${ }^{28}$ Steadman Jones (wie Anm. 19), 241-261.

${ }^{29}$ Marcel Mauss, Essai sur le don. Forme et raison de l'échange dans les sociétés archaïques, in: L'Année Sociologique, 1, 1923, 30-186 ; Claude Lévi-Strauss, Les Structures élémentaires de la parenté. Paris 1949.

${ }^{30}$ Alan Kidd, Philanthropy and the 'social history paradigm', in: Social History, 2, 1996, Nr. 2, 180-192, $186-187$.

${ }^{31}$ Fernand Braudel, The Mediterranean and the Mediterranean World in the Age of Philip II, Bd. 2, Berkeley 1996 [französische Erstveröffentlichung 1949], 826.

32 Zur Entwicklung dieses sozialpolitischen Ansatzes, vgl. Neil Gilbert/Barbara Gilbert, The Enabling State: Modern Welfare Capitalism in America. New York 1989.

${ }^{33}$ Anne Digby, British Welfare Policy: Workhouse to Workfare. London 1989, 85. Eine klare und prägnante Einführung zur Wohlfahrtsgeschichte in diesem Sinne findet sich bei John Stewart, The Mixed Economy of Welfare in Historical Context, in: Martin Powell (Ed.) Understanding the Mixed Economy of Welfare. Bristol 2007, 23-40.

${ }^{34} \mathrm{Vgl}$. David Wright/Anne Digby (Eds.), From Idiocy to Mental Deficiency: Historical perspectives on people with learning difficulties. London 1996; Peter Bartlett/David Wright (Eds.), Outside the walls of the asylum: the history of care in the community 1750-2000. London 1999.

${ }^{35}$ Graham Chester/Pamela Dale, Institutional Care for the Mental Defective, 1914-1948: Diversity as a Response to Individual Needs and an Indication of Lack of Policy Coherence, in: Medical History, 51, 2007, Nr. 1, $59-78$.

${ }^{36}$ Richard Trainor/Robert Morris, Preface, in: Robert Morris/Richard Trainor (Eds.), Urban Governance: Britain and Beyond since 1750. Aldershot 2000, xi.

${ }^{37}$ Vgl. z.B. Finlayson, (wie Anm. 1); Derek Fraser, The Evolution of the British Welfare State: A History of Social Policy since the Industrial Revolution. 3. Aufl. Basingstoke 2003; Bernard Harris, The Origins of the British Welfare State: Society, State and Social Welfare in England and Wales, 1800-1945. London 2004; Kathleen Jones, The Making of Social Policy in Britain: From the Poor Law to New Labour. 3. Aufl. London 2000.

${ }^{38}$ Pat Thane/Tanya Evans, Sinners? Scroungers? Saints? Unmarried Motherhood in Twentieth-Century England. Oxford 2012.

${ }^{39}$ Robert Putnam, Bowling Alone: The Collapse and Revival of American Community. New York 2000. Beispiele für den neuen Fokus auf das Vereinswesen finden sich bei Peter Ackers/Alastair J. Reid (Eds.), Alternatives to State-Socialism in Britain: Other Worlds of Labour in the Twentieth Century. Basingstoke 2016.

${ }^{40}$ Vgl. z.B. Pat Starkey, Families and Social Workers: The Work of Family Service Units, 1940-1985. Liverpool 2000.

${ }^{41}$ Georgina Brewis, A Social History of Student Volunteering: Britain and Beyond, 1880-1980. Basingstoke 2014; Caitriona Beaumont, Housewives and Citizens: Domesticity and the Women's Movement in England, 1928-1964. Manchester 2013.

${ }^{42}$ Vgl. z.B. Robert John Morris, Class, Sect and Party. The Making of the British Middle Class. Leeds, 1820-1850. Manchester 1990.

${ }^{43}$ Vgl. z.B. Bob Snape, Voluntary Action and Leisure: An Historical Perspective, 1830-1939, in: Voluntary Sector Review, 6, 2015, Nr. 2, 153-172; Laura Balderstone, Semi-detached Leicester: Social and Cultural Connections in Suburban Leicester, in: Richard Rodger/Rebecca Madgin (Eds.), Leicester: A Modern History. Lancaster 2016. 
${ }^{44}$ Seth Koven, Remembering and Dismemberment: Crippled Children, Wounded Soldiers, and the Great War in Great Britain, in: American Historical Review, 99, 1994, Nr. 4, 1167-1202; ders., Slumming: Sexual and Social Politics in Victorian London. Princeton 2004. Eine wohlwollendere Sicht dieser Form der visuellen Kultur findet sich bei Eloise Moss/Charlotte Wildman/Ruth Lamont/Luke Kelly, Rethinking Child Welfare and Emigration Institutions, 1870-1914, in: Cultural and Social History, 14, 2017, Nr. 5, 647-668.

${ }^{45}$ Vivienne Richardson, Clothing the Poor in Nineteenth-Century England. Cambridge 2013; Lucinda MatthewsJones, Lessons in Seeing: Art, Religion and Class in the East End of London, 1881-1898, in: Journal of Victorian Culture, 16, 2011, 385-403.

${ }^{46}$ Alan Kidd, Philanthropy and the Social History Paradigm, in: Social History, 2, 1996, Nr. 2, 180-192, $191-192$.

${ }^{47}$ Pat Starkey, Retelling the Stories of Clients of Voluntary Social Work Agencies in Britain after 1945, in: Anne Borsay/Peter Shapely (Eds.), Medicine, Charity and Mutual Aid: The Consumption of Health and Welfare in Britain, c.1550-1950. Abingdon 2007, 245-262.

48 John Pemble, So Very Silent, in: London Review of Books, 34, 25. Oktober 2012, Nr. 20, 10-12.

${ }^{49}$ Helen Rogers, Kindness and Reciprocity: Liberated Prisoners and Christian Charity in Early Nineteenth-Century England, in: Journal of Social History, 47, 2014, Nr. 3, 721-745.

${ }^{50}$ Virginia Crossman, Writing for Relief in Late Nineteenth-Century Dublin: Personal Applications to the Mansion House Fund in 1880, in: Cultural and Social History, 14, 2017, Nr. 5, 583-598. Zu den Armengesuchen als Quelle, vgl. z.B. Thomas Sokoll, Source Criticism: Credibility, in: Sokoll (Ed.), Essex Pauper Letters 1731-1837. Oxford 2001, 67-70; Peter Jones/Steven King (Eds.), Obligation, Entitlement and Dispute under the English Poor Laws, 1600-1900. Newcastle 2015.

${ }^{51}$ Thomas Carlyle, Past and Present. London 1843, Kapitel 6; vgl. ders., Chartism. London 1840.

52 Karl Marx/Friedrich Engels, Manifest der kommunistischen Partei. London 1848, 5. Zu Marx' sehr viel komplexeren und nuancierteren Geldphilosophie Suzanne de Brunhoff, Marx on Money. New York 1976.

${ }^{53}$ Vgl. Emile Durkheim, De la division du travail social. Paris 1893.

${ }^{54}$ Anthony Giddens, The Consequences of Modernity. Stanford 1990, 21-25.

${ }^{55}$ Wegweisend hier Deborah Valenze, The Social Life of Money in the English Past. Cambridge 2006.

${ }^{56}$ Sean O'Connell, Credit and Community: Working-Class Debt in the UK since 1880. Oxford 2009, 3.

57 Georg Simmel, Philosophie des Geldes. Berlin 1900, 88, 206.

${ }^{58} \mathrm{Zu}$ Webers Denken vgl. Bruce Mazlish, A New Science: The Breakdown of Connections and the Birth of Sociology. Oxford 1989, 217-240.

${ }^{59}$ Zu Parsons Denken vgl. Geoffrey Ingham, The Nature of Money. Cambridge 2004, besonders Kapitel 3: Money in Sociological Theory.

${ }^{60}$ Brian Harrison, Peaceable Kingdom: Stability and Change in Modern Britain. Oxford 1983, 217-259; Prochaska, Voluntary Impulse (wie Anm. 16).

${ }^{61}$ Colin Jones, Some recent trends in the history of charity, in: Martin Daunton (Ed.), Charity, Self-Interest and Welfare in the English Past. London 1996, 43.

62 Viviana A. Zelizer, Economic Lives: How Culture Shapes the Economy. Princeton 2011, 383. 
63 Peter Shapely, Charity, Status and Parliamentary Candidates in Manchester: A Consideration of Electoral and Charity Fields and the Social Basis of Power, 1832-1910, in: International Review of Social History, 44, 1999, Nr. 1, 1-21, 1; ders., Charity and Power in Nineteenth-Century Manchester. Manchester 2000.

${ }^{64}$ Mark Granovetter, Economic Action and Social Structure: The Problem of Embeddedness, in: American Journal of Sociology, 91, 1985, Nr. 3, 482.

${ }^{65}$ Karl Polanyi, The Great Transformation. Boston, MA, 1957 [Erstausgabe 1944], 57.

${ }^{66}$ Mark Granovetter, Society and Economy: Framework and Principles. Harvard 2017.

${ }^{67}$ Viviana A. Zelizer, Morals and Markets: The Development of Life in Insurance in the United States. New York 1979; dies., Pricing the Priceless Child. Princeton 1985.

${ }^{68}$ Dies., The Social Meaning of Money: Pin Money, Paychecks, Poor Relief, and Other Currencies. New York, 1994, 18.

${ }^{69}$ Zur Gründung der frühen voluntary hospitals im 18. Jahrhundert vgl. Roy Porter, The gift relation: philanthropy and provincial hospitals in eighteenth-century England, in: Lindsay Granshaw/Roy Porter (Eds.), The Hospital in History. London 1989. Eine Einführung ins System der voluntary hospitals in Großbritannien gibt John Woodward, To Do the Sick No Harm: A Study of the British Voluntary Hospital System to 1875. London 1974.

70 Zur Entwicklung britischer voluntary hospitals als Zentren medizinischer Behandlung und Bildung in internationaler Perspektive vgl. Daniel Fox, Health Policies, Health Politics: The British and American Experience, 1911-1945. Princeton 1986; Thomas Neville Bonner, Becoming a Physician: Medical Education in Britain, France, Germany, and the United States, 1750-1945. Oxford 1995. Zu voluntary hospitals innerhalb dieser mixed economy im Gesundheitswesen, siehe John Pickstone, Medicine and Industrial Society: A History of Hospital Development in Manchester and Its Region. Manchester 1985; Barry Doyle, The Politics of Hospital Provision in Early Twentieth-Century Britain. London 2014.

${ }^{71}$ Eine eingehende Diskussion zum Wesen und Gründen dieser Veränderungen findet sich bei George Campbell Gosling, Payment and Philanthropy in British Healthcare, 1918-48. Manchester 2017.

72 John Pickstone, Medicine and Industrial Society: A History of Hospital Development in Manchester and Its Region. Manchester 1985, 259.

73 Paul Starr, The Social Transformation of American Medicine. New York 1982, 159.

${ }^{74}$ Eine Erläuterung und Aufschlüsselung dieser Zahlen findet sich bei Gosling (wie Anm. 71), Kapitel 4: MiddleClass Medicine.

${ }^{75}$ Steven Cherry, Beyond National Health Insurance. The Voluntary Hospitals and Hospital Contributory Schemes: A Regional Study, in: Social History of Medicine, 5, 1992, Nr. 2, 455-482.

76 Martin Gorsky/John Mohan/Tim Willis, Mutualism and Health Care: Hospital Contributory Schemes in Twentieth-Century Britain. Manchester 2007, 65, 108.

77 Bristol Royal Infirmary, Report for 1921, 20.

78 Mehr Details zur Funktion des Bezahlungssystems für Krankenhausfürsorger, siehe Gosling (wie Anm. 71), Kapitel 3: Payment and the Sick Poor.

${ }^{79}$ Zum Beispiel bei Steven Cherry, Accountability, Entitlement, and Control Issues and Voluntary Hospital Funding c.1860-1939, in: Social History of Medicine, 9, 1996, 215-233. 
${ }^{80}$ George Campbell Gosling, The Birth of the Pregnant Patient-Consumer? Payment, Paternalism and Maternity Hospitals in Early Twentieth-Century England, in: Jennifer Evans/Ciara Meehan (Eds.), Perceptions of Pregnancy from the Seventeenth to Twentieth Century. London 2017.

${ }^{81}$ Mehr Details liefert die Diskussion der ,Klassendifferenzierung‘ bei Gosling (wie Anm. 71).

82 Siehe Mary Fissell, Patients, Power, and the Poor in Eighteenth-Century Bristol. Cambridge 1991, 7, $12,74$.

${ }^{83}$ Mehr Details liefert die Diskussion ,wirtschaftlicher Gegenseitigkeit' bei Gosling, (wie Anm. 71).

${ }^{84}$ Alan Kidd, Philanthropy and the Social History Paradigm, in: Social History, 2, 1996, Nr. 2, 180-192, 184. 